\title{
Nuevas combinaciones y nuevos sinónimos en especies de Brasil de Diodia s. lat. (Spermacoceae - Rubiaceae)
}

New combinations and synonyms in species of Diodia s. lat. (Spermacoceae - Rubiaceae) from Brazil

\author{
Elsa L. Cabral ${ }^{1,2} \&$ Andrea A. Cabaña Fader ${ }^{1}$
}

\begin{abstract}
Resumen
Durante la actualización de la lista de especies de la tribu Spermacoceae (Rubiaceae) de Brasil se realizaron algunas consideraciones nomenclaturales para cinco nombres publicados de Diodia. Se presentan dos nuevas combinaciones: Diodella angustata (Steyerm.) E.L. Cabral \& A. Cabaña-Fader y Mitracarpus froesii (Sucre) E.L. Cabral \& E.B. Souza. Se incluye a Diodia barbeyana como nuevo sinónimo de Diodella gardneri, se cita a Diodia microcarpa como nuevo sinónimo de Borreria palustris y a D. appariciana como nuevo sinónimo de Borreria multiflora.
\end{abstract}

Palabras clave: Flora do Brasil, taxonomía, Diodella, Mitracarpus.

\begin{abstract}
While updating the list of species of the tribe Spermacoceae (Rubiaceae) from Brazil, the nomenclature of five published Diodia names was examined. We present two new combinations Diodella angustata (Steyerm.) E.L. Cabral \& A. Cabaña-Fader and Mitracarpus froesii (Sucre) E.L. Cabral \& E.B. Souza. Diodia barbeyana is included as new synonym of D. gardneri, Diodia microcarpa is cited as new synonym of Borreria palustris, and $D$. appariciana as a new synonym of Borreria multiflora.
\end{abstract}

Kew words: Brazilian flora, taxonomy, Diodella, Mitracarpus.

\section{Introducción}

Diodia L. es un género americano perteneciente a la tribu Spermacoceae. Fue descrito por Linneaeus (1753) sobre $D$. virginiana, posteriormente fueron incorporadas varias especies de caracteres morfológicos diferentes a la especie tipo, razón por la cual se ha convertido por varios años en un género conflictivo de difícil interpretación. Bacigalupo \& Cabral (1999) realizaron la revisión de Diodias. str. y reconocieron para este género sólo a cinco especies de hábito rastrero que viven en ambientes palustres, con escasas flores axilares, de corola con tubo estrecho y largo, estilo bífido y frutos totalmente indehiscentes. Con la delimitación de este género, se segregaron a las especies que no se ajustaban al concepto de dicho género siendo reordenados en los siguientes taxones: Diodella Small, Galianthe Griseb. subg. Ebelia E.L. Cabral \& Bacigalupo y Borreria G. Mey. Subg. Dasycephala Bacigalupo \& E.L. Cabral.
Durante la actualización del listado de especies de la tribu para Brasil (Cabral \& Salas 2010), se encontraron otros taxones publicados como Diodia que no se correspondían a la descripción del género. De la consulta de descripciones originales y el análisis de los correspondientes ejemplares tipo, se transfieren dichas especies a los siguientes taxones.

Diodia angustata Steyerm. se asimila a Diodella Small (Diodella angustata (Steyerm.) E.L. Cabral \& A. Cabaña-Fader) y Diodia froesii Sucre al género Mitracarpus Zucc. (Mitracarpus froesii (Sucre) E.L. Cabral \& E.B. Souza). Se discute la identidad de Diodia barbeyana Huber, que aquí se incluye como sinónimo de Diodella gardneri (K. Schum.) Bacigalupo \& E.L. Cabral. Se proponen dos nuevos sinónimos: Diodia microcarpaK. Schum. ex Glaz. de Borreria palustris (Cham. \& Schltdl.) Bacigalupo \& E.L. Cabral y a $D$. appariciana Rizzini de $B$. multiflora (DC.) Bacigalupo \& E.L. Cabral.

\footnotetext{
Instituto de Botánica del Nordeste (UNNE-CONICET), Facultad de Ciencias Exactas y Naturales y Agrimensura, UNNE, Casilla de Correo 209, 3400 Corrientes, Argentina.

22Autor para correspondencia: ecabral@agr.unne.edu.ar, ferber@arnet.com.ar
} 


\section{Resultados y Discusión}

\section{Nuevas combinaciones}

Diodella angustata (Steyerm.) E.L. Cabral \& A. Cabaña Fader, comb. nov. Diodia angustata Steyerm., Contrib. Sci. Los Angeles County Mus. 21: 26, fig. 13-14. 1958. Tipo: BRASIL. GOIÁS: Region of the Chapada dos Veadeiros, on grassy hillslope near canyon bottom in sandstone area $14 \mathrm{~km}$, south of Veadeiros, at $14^{\circ} 30^{\prime}$ 'S, $47^{\circ} 30^{\prime} \mathrm{W}$, 24.IV.1956, fl., E.Y. Dawson 14672 (holotipo R!; isotipos F, LAM, US!).

Esta especie se caracteriza por ser un sufrútice erecto, de tallo simple o con ramificación distal, con pelos adpresos cinéreo-hispídulos. Hojas sésiles, 2,5-6 × 0,2-0,8 cm, linear-lanceoladas, glabras o pubérulas hacia la base, 4 o 5 pares de nervios secundarios visibles en el envés. Inflorescencia apical, pauciflora. Flores con cáliz e hipanto hispídulos, corola infundibuliforme, externamente hirsuta y glabra por dentro, excepto un anillo de pelos en el tercio inferior del tubo. Estigma capitado-bilobado.

Hasta el presente sólo se conoce el material tipo de Brasil, estado de Goiás, Chapada dos Veadeiros.

Se propone esta nueva combinación por haber observado en el ejemplar tipo los siguientes caracteres que justifican transferir Diodia angustata al género Diodella: inflorescencia en glomérulos paucifloros (vs. 1 o 2 flores axilares incluidas en la vaina estipular), flores con corola infundibuliforme (vs. flores con tubo largo y estrecho), interior de la corola con anillos de pelos cerca de la base (vs. pelos en los lóbulos), estigma capitado-bilobado (vs. estilo con ramas estigmáticas filiformes).

Mitracarpus froesii (Sucre) E.L. Cabral \& E. B. Souza, comb. nov. Diodia froesii Sucre, Rodriguésia 38: 254, fig. 5. 1971. Tipo: BRASIL. AMAZONAS: Boa Vista, Río Araçá, subafluente do Río Negro, terra firme, baixa, 24.X.1952, R.L.Fróes \& G. Addison 29062 (holotipo IAN!).

Esta especie se reconoce por ser un sufrútice con hojas de 20-80 × 4-9 mm, linear lanceoladas, glabras; glomérulos axilares numerosos. Cáliz con 4 segmentos desiguales, ciliados; corola tubulosa externamente glabra, pilosa en el ápice de los lóbulos; estambres fijos en la garganta de la corola, estigma bífido. Fruto globoso, de dehiscencia transversal, pubérulo en la mitad superior, con sépalos persistentes muy desarrollados, formando un tubo calicino notable. Semilla con depresión cuadrangular en la cara ventral

Se conoce hasta el momento solo el ejemplar tipo del Estado de Amazonas.
Esta especie se asemeja a Mitracarpus hirtus (L.) DC. por ser un sufrútice erecto, con numerosos glomérulos axilares, pero se diferencia de la misma porque las semillas tienen la cara ventral con depresión en forma de X.

\section{Nuevos sinónimos}

Diodella gardneri (K. Schum.) Bacigalupo \& E.L. Cabral, Darwiniana 44(1): 98, fig. 1. 2006. Diodia gardneri K. Schum. in Martius, Fl. bras. 6(6): 402. 1889. Borreria gardneri (K. Schum.) Bacigalupo \& E.L. Cabral, Opera Bot. Belg. 7: 307. 1996. Tipo: BRASIL. CEARÁ: Habitat in collibus arenosis prope Aracay, G. Gardner 1705 (holotipo B, destruido; isotipo BM!).

Diodia barbeyana Huber, Bull. Herb. Boissier ser. 2(1): 325. 1901. Tipo: BRASIL. CEARÁ: Fortaleza, entre os arbustos na planície ao sul da capital, 1897 , J.E. Huber 28 (holotipo RB!), syn. nov.

Esta especie es frecuentemente confundida en los herbarios con Diodella radula (Willd. ex Roem. $\&$ Schult.) Delprete, de la cual se diferencia por el hábito escandente (vs. hábito decumbente a postrado), por la semilla ruminada, con surcos transversales en el borde de la cara ventral y surcos profundos que se extienden hasta el borde superior de la semilla ( $v s$. semilla sin surcos en la cara ventral), sépalos de 5-6 $\mathrm{mm}$ long. de borde piloso y dorso glabrescente ( $v s$. sépalos de 1,5-4,2 $\mathrm{mm}$ pubescentes).

Vive entre 600-1200 m.s.m, en la caatinga o en la transición caatinga-cerrado en el nordeste de Brasil, en los estados de Bahia, Ceará y Piauí.

Stafleu \& Cowan (1983) mencionan que la colección original de J. E. Huber estaba depositada en el herbario MG, sin embargo las colecciones más antiguas de este herbario fueron llevadas por Adolfo Ducke a RB aproximadamente en la segunda década del siglo XX (Ricardo Secco, com. pers.). Recientemente se confirmó que este material efectivamente se encuentra depositado en dicho herbario RB (Rafaela Forzza, com. pers.).

Bacigalupo \& Cabral (2006). mencionan que sólo se conocía el ejemplar tipo del estado de Ceará. Con el material aquí analizado se amplia el área de distribución a los estados de Bahia y Piauí. Material adicional examinado: BRASIL. BAHIA: Morro do Chapéu, 26.IX.1965, A.P. Duarte 9196 (RB); 1141'34”'S, 44¹1'56”'W, 30.II. 2005, E.B Souza et al. 1058 (HUEFS); Umburanas, Serra do Curral Feio a beira do rio Tabuleiro, 10²1'S, 41 ${ }^{\circ} 13^{\prime}$ 'W, 12.IV.1999, L.P. Queiroz et al. 5427 (HUEFS). CEARÁ: Fortaleza, Cajazeiro, 1.VI.1960, Matos 616 (RB); Av. Perimetral, praia do Futuro, 6.VI.1960, Matos 600 (RB). PIAUÍ: Castelo do Piauí, 5¹9’20”S, 41³3’09”'W, 13.VI.1995, M.S. Bona Nascimento et al. 1053 (CTES). 
Borreria palustris (Cham. \& Schltdl.) Bacigalupo \& E.L.Cabral,Hickenia 2: 264, fig. 2. 1998. Diodia palustris Cham. \& Schltdl.,Linnaea 3: 347. 1828. Tipo: BRASIL. Ad fretum St. Catharinae Brasiliae aequinoctialis, $F$. Sellow s.n. (holótipo B destruido). Neótipo: designado por Bacigalupo \& Cabral (1998): BRASIL. RIO GRANDEDOSUL: Morro da Polícia, pr. Porto Alegre, 14. III. 1949, B. Rambo 40486 (LIL!).

Diodia microcarpa K. Schum. ex Glaz., Bull. Soc. Bot. France 56 (Mém. 3d): 361. 1909. BRASIL. "Brasília", A.F.M. Glaziou, 18283 (holótipo B destruído, foto F 867!), syn. nov.

Esta especie se caracteriza por presentar un hábito subpostrado con nudos radicantes, tallos tetrágonos alados con papilas retrorsas. Hojas 1,5-4×0,8-2 cm, elípticas u obovales con base atenuada en pseudopecíolo, impreso-nervosas. Inflorescencias tirsoides con inflorescencias parciales glomeriformes. Flores 2(-3)-partido, corola 2-3(-4)-lobada, estilo bífido o trífido. Fruto con mericarpos indehiscente y semillas surcadas en la cara ventral, alrededor del estrofíolo.

Habita en terrenos bajos, anegadizos y pantanosos. Tiene una amplia distribución en América del Sur y en Brasil vive desde Bahia a Rio Grande do Sul.

Borreria multiflora (DC.) Bacigalupo \& E.L. Cabral, Opera Bot. Belg. 7: 297. Diodia multiflora DC. Prodr. 4: 564. 1830. Tipo: BRASIL. "In Brasilia", 1828, J.B.E. Pohl s.n. (holotipo G-DC.!).

Diodia appariciana Rizzini, Rev. Bras. Biol. 7: 277. 1947. Tipo: BRASIL. MINAS GERAIS: Carandaí, 18.II.1946, A.P. Duarte 532 (holotipo RB!), syn.nov.

Esta especie se reconoce por ser un sufrútice escandente de 1-2 m alt. con tallos tetrágonos pubescentes. Hojas de 15-55 ×5-13 mm, elípticasoblongas o obovadas, impreso-nervosas, escábridas, coriáceas. Glomérulos terminales y 20-25 axilares. Flores e hipanto escabrosos, corola 3,5-4 mm long., estambres 4 excertos, estigma bífido. Fruto con dos mericarpos indehiscentes y semilla finamente reticulada.

Habita en terrenos bajos, arenosos. Es ampliamente distribuida en América del Sur; en Brasil vive desde Goiás, Distrito Federal, Mato Grosso, Mato Grosso do Sul y San Pablo.

\section{Agradecimientos}

Agradecemos a los Dr. Ricardo Secco y Dra. Rafaela Forzza por la gentileza en respondernos a nuestras consultas, a Roberto M. Salas por sus valiosas sugerencias y a Luciano Margalho por su amabilidad al enviarnos con rapidez los fototipos.

\section{Referencias}

Bacigalupo, N.M. \& Cabral, E.L. 1999. Revisión de las especies americanas del género Diodia (RubiaceaeSpermacoceae). Darwiniana 37: 153-165.

Bacigalupo, N.M. \&. Cabral, E.L. 2006. Nuevas combinaciones en el género Diodella (RubiaceaeSpermacoceae). Darwiniana 44: 98-104.

Cabral, E. \& Salas, R. 2010. Diodella. In: Lista de Espécies da Flora do Brasil. Disponible en: <http:/ /floradobrasil.jbrj. gov.br/2010/FB013931>. Acceso a mayo 2010 .

Linnaeus, C. 1753. Diodia. Species Plantarum. 1:104 Holmiae.

Stafleu, F.A. \& Cowan, R.S. 1983. Taxonomic literature 2. 2nd ed. Scheltema \& Holkema, Bohn, Utrecht. Pp. 353-354. 